\title{
(2) \\ Using data exploration to help marketers: How Internet sites can learn from telephone companies
}

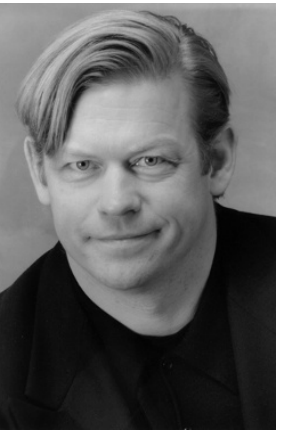

Received (in revised form): 25th February, 2000

\section{John K. Thompson}

vice president, worldwide marketing, WhiteCross Systems joined WhiteCross in October 1999 and is responsible for all global marketing activities from branding and image to marketing programmes. He has over 15 years' experience spanning all major technology management functions for high technology organisations.

In his current role, he formulates and executes the strategic direction for WhiteCross. His technology expertise includes knowledge discovery, analytical applications, data exploration, data warehousing and database systems. Prior to joining Whitecross he held a number of senior technology and marketing positions at Magnify, Inc., PLATINUM Technology, IBM and Metaphor Computer Systems. John holds a BSc from Ferris State University and an MBA in Marketing from DePaul University.

Abstract The Internet and e-business offer excellent opportunities for gathering customer data and companies can use these data within marketing programmes highly effectively. Potential activities range from simple tasks such as direct mail to complete reinvention of a business by changing product lines based on customer preferences. Many companies, however, are not equipped to perform the large scale data analysis required to achieve these marketing objectives. Using the telecommunications industry as an example, this paper will outline best practices in data analysis and explore some of the ways in which these data can be used. Telecommunications companies have faced the large volumes of data in the form of call logs that companies moving on to the Internet are now experiencing through visits to their websites.

\section{INTRODUCTION}

Many organisations now employ advanced technology as a matter of course to develop highly effective, precisely targeted marketing campaigns. Segmenting and analysing customer databases enable companies to identify precise behavioural patterns of customers and tailor products to suit individual requirements.

In the majority of industries, the amount of data collected is manageable but in the utilities sector, the volumes of data collected are immense. In the case of telecommunication companies, each call is detailed, explaining who made it, who was called, duration and time of day. Every single call record is then stored in a database. Utility companies now create data warehouses that can be measured in terabyte terms. This level of customer data is no longer unique to the utilities industry as a result of website data. Companies can collect incredible volumes of data about customers visiting a website. Although this presents excellent opportunities for increased customer understanding and, therefore, target marketing, it is also a challenge for businesses to adapt their database marketing processes to deal with exponential amounts of data. By looking at an example from the telecoms industry, it is easier to understand how to 
employ this new business model. This paper discusses what data analysis can be used for in both telecoms and e-business and best practices to gain competitive advantage.

\section{USING DATA ANALYSIS FOR COMPETITIVE ADVANTAGE}

The main method of obtaining market share in the telecoms sector is customer segmentation. Deregulation has led to increasing competition but there are very few areas for differentiation. Almost every consumer in Britain uses the telephone and expects their service to work perfectly all the time. Itemised billing and efficient, courteous customer services are also standard expectations. Companies are looking to determine who their most profitable customer segments are and then develop tailored packages that will ensure that they remain both competitive and profitable. They also need to find out where they are performing badly so they can rectify any failings and they also need to identify and gain a share of growth areas of the market. Identifying ways to reach new customers is therefore also important. Because the market is changing rapidly it is inadvisable for companies to focus only on one or two segments of their market, as this will make them vulnerable.

Telecoms companies are meeting these challenges through sophisticated data analysis techniques. Joining lifestyle, billing and customer relationship management (CRM) data with call detail is providing a potent weapon for telecoms companies (telcos) focused on improving customer acquisition and/or care and retention.

While product purchases, lifestyle information and billing data provide important information about customers, it is call detail records that describe a customer's behaviour and define their satisfaction with the services offered. Call detail records describe the transactions between customer and telco. The value of a customer to a telco is directly related to the value/frequency of the transactions undertaken. The challenges for a telco are increasing the frequency of its highest valued transactions while protecting the purchasers of these transactions from its competitors. These challenges require a complete understanding of a customer's need for and satisfaction with a particular service, and knowledge of any barriers to use, eg poor quality of service, lack of availability. The key to success is monitoring and analysing usage - call pattern analysis derived from call detail records. This provides the only true and reliable measure of how a customer values a service.

In the author's experience at WhiteCross, telcos have found data analysis invaluable. It can be used to:

- perform 'train-of-thought' analysis on large volumes of call detail, it scales to billions of rows per second

- handle complex joins between different data source, eg call detail, lifestyle and billing data, input from CRM systems and third party feeds

- react rapidly to changes in new or additional data by making data 'analysis-ready' through a concurrent load and go approach to data extraction, transformation and loading.

Telcos can therefore build and deploy:

- early warning systems

- behavioural models for re-pricing, product and new plan design

- segmentations for targeting, churn and fraud management.

This collating and analysis of information 
enables telcos to be prepared for competitive landscape and market fluctuations, to create tailored cost effective promotions and packages based on full volume call detail data, to identify customers likely to leave the network and to detect fraud. It is a sophisticated and thorough process that is part of every business decision made within the organisation.

\section{APPLYING DATA ANALYSIS TO ENABLE BUSINESS BENEFITS}

Telcos typically use data analysis in all aspects of their business from developing new campaigns to fraud detection. The following are typical examples:

- calling behaviour: eg analysis of calling patterns; length, destination and timing of calls; before and after call volume measurement for campaigns; future calling pattern prediction for product planning

- pricing: eg measuring the impact of pricing on calling behaviour, developing models for optimisation of pricing, analysis of re-pricing impact, profitability by customer/day/time

- campaign targeting: eg segmentation, special offer analysis, identification of most/least profitable customers, identification of up-selling and cross-selling opportunities

- churn: eg building profiles of normal usage to predict future customer behaviour and to flag patterns likely to predict churn, identifying loyal customer patterns and the impact of enticements

- network performance: eg root cause analysis and service level

measurement, optimisation of network usage, outgoing/incoming call analysis, network cost allocation, and 'hotspot' cell location

— fraud: real time and subscription fraud detection. Unusual and unrealistic calling patterns can be quickly detected.

\section{WHY ANALYSE THE ENTIRE DATABASE?}

One option for telcos and other industries collecting large volumes of data is to make decisions based on analysis of samples of the data rather than the whole database, enabling the company to gain an insight into a representative section of customers, but with less effort. There are, however, some good reasons why telcos should not use this approach, the primary reason being that it is not possible to gain the information outlined above by sampling.

The problem with sampling is that a distorted picture can appear and with such tight profit margins a mistake of one penny per call or per unit can be very costly. The only reason that companies resort to sampling is because their data analysis system is not powerful or scaleable enough to analyse all their data, which is why WhiteCross decided to build a system that provides unlimited scalability and fast response times.

The limitations of sampling are:

- detail gets closer to the truth, it represents 'facts' whereas samples can only be a best guess of the truth

- rare events undermine general purpose samples. For example: long tail duration curves; data niches perhaps while characterising behaviour patterns or drilling into the data; small, high-value segments such as top 100 customers can be missed or over-represented

- slice and dice operations, so common in business intelligence operations, are undermined

- certain analyses are impossible, eg top called numbers such as looking for 
calling neighbourhoods could not be analysed: if CustB is in the top 10 called numbers of Cust $A$, then is CustA in CustB's top 10?

- aggregation operations, often used alongside sampling to compact data, presume the results in advance. For example 'date' could be aggregated in many ways: onto day of week, date of month, month of year, week of year etc. Only the ongoing analysis of the business problem will determine which is the most suitable, to have made this choice a priori limits the insight that the data can reveal

- specific purpose samples need considerable time (eg weeks) plus computing power to prepare. This conflicts greatly with business needs. Therefore, the natural tendency to try to avoid the delays surrounding construction of new samples will favour getting fast erroneous results - samples cannot be reliably used by business professionals, they require the skills of statisticians. All results must be verified 'after the analysis' to ensure that the sample is representative of phenomena being described - that the conclusions are solid and not based on some sampling artefact. This amounts to 'train of misconception' (if such checking is missed out) or 'slow train of statistics' (if such checking is done rigorously)

- considerable statistical skill is required to manage a sample-based project correctly — it is not possible to predict the point in the analysis at which a sample would be unrepresentative. As such there needs to be continual vigilance to ensure the statistical accuracy of any results

- updating or refreshing the data is a more complex issue, not only does the sampling step need to be undertaken again, the validity of the sample must also be re-assessed with every refresh of the data. This is in contrast to the simplicity of taking the raw calls and re-analysing the data.

It is only by analysing the entire database that rapid development of targeted offers and ensuring that they reach the right consumers become possible. Direct mail and advertising budgets are not wasted on the wrong people and the company can accurately predict whether a special offer or plan will be profitable for them based on the number of people likely to take up an offer.

\section{CABLE \& WIRELESS: PUTTING THEORY INTO PRACTICE}

Cable \& Wireless use WhiteCross Data

Exploration Server (WX/DES) to develop these types of predictions. For example, by measuring the impact on the consumer of changing mobile call prices they could check whether specific groups of customers would be adversely affected and, therefore, how the company's profit margins would fare. They were able to decide whether to institute the changes with confidence as the decision was based on real and full customer data.

Because the WX/DES provides an immediate response to queries, special offers can quickly be adapted if data analysis demonstrates that an offer will not be as profitable as expected. For example Cable \& Wireless offered two hours of call time to Australia for f5.00. The company needed to predict the financial impact. If everyone stayed on the phone for two hours it would not be a profitable offer for Cable \& Wireless, but by conducting data analysis the company could predict that the offer would seem limitless to customers but in actual fact most of them could not talk for that long. If it emerged that people were in fact using the system for too long the offer would not be repeated or extended. 
Price elasticity has been examined by running a series of very short campaigns and monitoring call behaviour before making longer-terms decisions. For example, cutting the price of calls to France did not stimulate more demand, but lowering the rates to New Zealand did. The company can identify which offers are profitable enough to be integrated indefinitely. For example, Cable \& Wireless kept the 50 pence on Saturdays offer running permanently.

Customer retention can also benefit. By using fine data segments, predictions can be made about which groups of customers are vulnerable to offers from competitors. Cable \& Wireless rang each of their customers that they believed were no longer on the most cost efficient calling plan to discuss moving to a new plan. The company is now able to offer a 'price pledge' as the central tenet of its customer proposition. It is a compelling and reassuring offer: 'You'll save money over BT including all their discount schemes or we'll give you double the difference back'.

The data analysis system has been highly cost effective and the savings resulting from one major analysis paid for one business unit's share of the system within six weeks.

The Cable \& Wireless team are planning to leverage the significant benefits of data analysis into other areas of their business, especially into new areas where there is not an operating history to draw upon. Detailed data analysis is planned for the new digital television offering. For example, analysing which films are ordered by tightly defined segments allows relevant movie offers to be made directly to interested customers. This analysis capability is also set to be instrumental in the Internet website traffic analysis, and in designing relevant call plans for customers who use the Internet.
Between 1998 and 1999, Cable \& Wireless Communications has turned a $£ 30 \mathrm{~m}$ loss into a $£ 140 \mathrm{~m}$ profit before tax. In relating this to the large-scale detailed analysis capability, Janet Somerville, marketing director of Cable \& Wireless said: "We could not have achieved that profit level without the right price plans in place, and we wouldn't have been able to construct those plans without the WhiteCross capability'.

\section{APPLYING THIS MODEL TO THE WEB}

The Cable \& Wireless story is a great example of using data analysis successfully and other organisations can benefit from this approach. It is not just telcos that have access to this kind of data about their customers - every company with a website has logs that can be analysed.

The majority of websites still measure their success through hits and page views and may also have site operators reviewing web logs and receiving reports from ad-tracking software, but few do even the simplest of analyses with the data. As sites become more sophisticated, some companies may build separate reports on the number of chat sessions, e-commerce transactions, or customer-support calls, but they do not have integrated systems for determining what the typical user is doing, what he or she finds appealing, or what he or she dislikes. ${ }^{1}$

By creating a data ensemble using information from website activity and other on-line and off-line customer data, such as subscription and purchase files, companies can understand better how to acquire and retain customers through their website and also how to evolve their website to meet customer requirements. The author's company manages the loading and analysis of many 
data sources so that companies can analyse their business on a continuum from logs to loyalty. Companies can gain insight about the stickiness of their site, seasonal trends, on- and off-line purchase behaviour and customer acquisition, loyalty and wallet share. Any company looking to acquire, understand and retain high-value users can use data analysis to:

- maximise on-line advertising premiums by attracting the right visitors and customers and also identifying where the adverts should be placed within a site and what rates each site area should command

- understand the preferences and interests of subscribers to optimise e-commerce revenues. This is particularly important for portals - profile customers that are likely to respond to off-line advertising and identify where these adverts should be placed.

Enterprises with an on-line presence may not have previously collated this amount of customer data through their traditional business model and are not equipped to take advantage of these data. They know they need to create an effective web presence to avoid losing market share to dot.coms but do not want to make expensive mistakes with their website or cannibalise their existing business. They therefore need to apply highly targeted marketing techniques, first to understand their web customer base and secondly to understand how they can improve areas of their website and provide more targeted solutions. For these companies it may be worthwhile for them to consider outsourcing in order to gain access to data analysis consultancy expertise.

Companies doing business on the Internet need to provide more competitive pricing to attract and retain customers. As well as reducing obvious overheads such as staff and office space, organisations are increasingly focused on making less profit per customer so that they attract more customers which will lead to increased market share and, therefore, increased profits. If a company gains more customers it can buy larger quantities of goods from its suppliers who will therefore provide a discount, which means that profitability per customer may stay the same or even increase, even though the cost per customer has decreased. Only data analysis of the entire customer database can help companies accurately predict this. Also, data analysis can be used when the offer or new pricing structure has been in place for just a short time in order to confirm the prediction and check profitability.

\section{CONCLUSION}

It is interesting to see how data analysis techniques can be taken from a major established industry and applied to the Internet, a medium still in its infancy. The data analysis techniques used by a major conglomerate such as Cable \& Wireless can also be used by an Internet start up and both can gain an equally dramatic impact on their business in terms of customer attraction and retention.

\section{Reference}

1 Schmidt, E. (1999) 'Measuring web success', Forrester Research. 